\title{
Back Translation
}

National Cancer Institute

\section{Source}

National Cancer Institute. Back Translation. NCI Thesaurus. Code C142397.

The process of translating a document back to it originally published language. 